\title{
Systemic Corticosteroids in Vitiligo
}

\author{
Binod K. Khaitan and Sushruta Kathuria \\ Department of Dermatology and Venereology \\ All India Institute of Medical Sciences, New Delhi \\ India
}

\section{Introduction}

Vitiligo is an acquired disorder characterized by depigmented macules on skin, hair, and mucosa. Its prevalence worldwide is $0.1-2 \%$ and varies from country to country. ${ }^{1}$ It is associated with high psychiatric morbidity and dysfunction, particularly in darker skin types where the contrast between normal and depigmented skin is highly pronounced.2,3 The usual approach to the treatment of vitiligo has been to regain pigment in vitiliginous areas rather than controlling the disease process. This is akin to treating the effects of the disease rather than treating the disease itself. Such an approach frequently results in frustration because patients can continue to develop new lesions at other sites even while existing lesions are repigmenting, or the disease may be reactivated at a later stage even after achieving a fair degree of therapeutic success. 4 Therefore, the important priority for the treatment of vitiligo should be first to control the disease process, and then taking measures to repigment existing lesions.

Systemic corticosteroids are a broad group of drugs that are widely used in various dermatological and non-dermatological conditions. Corticosteroids, both topical and systemic, have been used in vitiligo for over four decades with some concern about its efficacy as well as its known or expected adverse effects. Further research may provide evidence that systemic corticosteroids may be an important modality for the treatment of progressive vitiligo.

\section{History}

In 1967, oral corticosteroids were used for the first time in vitiligo. Triamcinolone orally was found to have a synergistic effect with methoxypsoralen as compared to methoxypsoralen alone. ${ }^{5}$ In 1976, adrenocorticotropic hormone (ACTH) was found to be effective in inducing repigmentation in patients who had previously failed photochemotherapy with psoralen followed by UVA exposure (PUVA). ${ }^{6}$ In the same year, a mixture of prednisolone, betamethasone, paramethasone acetate and methylprednisolone given orally was effective in producing satisfactory repigmentation in one-third of patients studied, thus confirming the efficacy of corticosteroids in vitiligo. ${ }^{7}$ Treatment of vitiligo is known to be long-term, hence the question of long term safety of systemic corticosteroids is an important consideration. To reduce long-term complications of corticosteroids, oral mini-pulse therapy was introduced in 1989 and was established in 1993, where instead of daily oral corticosteroids, a long-acting 
corticosteroid was given on two consecutive days of the week.8,9 Since then, systemic corticosteroids are used in day-to-day practice in extensive and progressive vitiligo.

\section{Structure and classification}

The structure of corticosteroids consists of three hexane rings and one pentane ring known as the cyclopentanoperhydrophenathrene nucleus. The different systemic corticosteroids are variations of this basic structure. Systemic corticosteroids are classified according to their half-lives. (Table 1)

\begin{tabular}{|c|c|c|c|c|c|}
\hline Corticosteroid & $\begin{array}{l}\text { Equivalent } \\
\text { dose (mg) }\end{array}$ & $\begin{array}{c}\text { Glucocorticoid } \\
\text { potency }\end{array}$ & $\begin{array}{c}\text { Mineralocorticoid } \\
\text { potency }\end{array}$ & \begin{tabular}{|c|} 
Plasma \\
half- life \\
(minutes)
\end{tabular} & $\begin{array}{c}\text { Biological } \\
\text { half-life } \\
\text { (hours) }\end{array}$ \\
\hline \multicolumn{6}{|l|}{ Short Acting } \\
\hline Cortisone & 25 & 0.8 & $2+$ & $30-90$ & $8-12$ \\
\hline Hydrocortisone & 20 & 1 & $2+$ & $60-120$ & $8-12$ \\
\hline Deflazacort & 6 & & & $90-120$ & $<12$ \\
\hline \multicolumn{6}{|l|}{ Intermediate Acting } \\
\hline Prednisone & 5 & 4 & $1+$ & 60 & $24-36$ \\
\hline Prednisolone & 5 & 4 & $1+$ & $115-212$ & $24-36$ \\
\hline Methylprednisolone & 4 & 5 & 0 & 180 & $24-36$ \\
\hline Triamcinolone & 4 & 5 & 0 & $78-188$ & $24-36$ \\
\hline \multicolumn{6}{|l|}{ Long Acting } \\
\hline Dexamethasone & 0.75 & $20-30$ & 0 & $100-300$ & $36-54$ \\
\hline Betamethasone & $0.6-0.75$ & $20-30$ & 0 & $100-300$ & $36-54$ \\
\hline
\end{tabular}

Table 1. Classification of Corticosteroids10,11

The structure, mechanism of action, and pharmacokinetic profile of various systemic corticosteroids are similar. The main difference is in their glucocorticoid and mineralocorticoid effects and biological half-life. Hence, the choice of corticosteroid is decided by the duration of action needed, the dosing schedule to be used (daily versus weekly) and the clinical profile of the patient. Having a working knowledge of equivalent dosing helps in dose conversion when one corticosteroid is switched to another.

\section{Mechanism of action of corticosteroids in vitiligo}

Vitiligo is considered to be primarily an autoimmune condition that results in the loss of melanocytes. Melanocytes are lost in vitiligo due to the destruction of melanocytes by humoral or intrinsic cellular mechanisms. ${ }^{2}$ Humoral mechanisms involve destruction of melanocytes by pathogenic autoantibodies directed against melanocytes and the tyrosinase enzyme. Cellular mechanisms are as important as humoral in destroying melanocytes. Biopsies from vitiligo lesions show mild mononuclear infiltrate in the margins of lesional skin in active vitiligo with or without basal vacuolization. Active lesions show increased numbers of CD4+ T cells and increased epidermal expression of ICAM-1. ${ }^{13}$ Peripheral T-cell activation is also seen in nonsegmental vitiligo and T-cell dysregulation is not only limited to the lesions. ${ }^{14}$ The peripheral blood of vitiligo patients express more of the cutaneous 
lymphocyte-associated antigen positive melanocyte-specific cytotoxic $\mathrm{T}$ lymphocytes indicating that there is recruitment of T cells in the circulation to the skin. ${ }^{15}$ Both the CD4+ and CD8+ T cells from perilesional and non-lesional skin in vitiligo show polarization towards the Type 1 cytokine profile which parallels depigmentation. ${ }^{16}$ The melanocyte-specific cytotoxic $\mathrm{T}$ cells found in perilesional biopsies have the ability to infiltrate normal skin and destroy the melanocytes. ${ }^{17}$ Besides cytotoxic $\mathrm{T}$ cells, regulatory $\mathrm{T}$ cells (T regs) also have a role in vitiligo. The $\mathrm{T}$ reg population is not decreased in the peripheral circulation, however, these $\mathrm{T}$ regs are not able to settle in the skin and this defect seems to be crucial in the observed perpetual antimelanocyte reactivity in progressive disease. ${ }^{18}$

Corticosteroids suppress autoantibody formation and likely induce apoptosis of cytotoxic $\mathrm{T}$ cells. The autoimmune hypothesis still prevails in the pathogenesis of non-segmental vitiligo, and the autoimmune process is not limited to the skin. In progressive disease, there is continuous assault on melanocytes. Topical corticosteroids, tacrolimus or any other topical agent may suppress autoimmune dysfunction at the site of a vitiligo lesion, but these do not have any effect on the disease process per se. Photochemotherapy and phototherapy stimulate melanocytes to cause repigmentation and have minimal immunomodulatory action on T-lymphocytes, but may not halt the rapid destruction of melanocytes. Systemic corticosteroids act not only on lesional immune activity, but also on the $\mathrm{T}$ cells in peripheral circulation. Besides modulating cell-mediated immunity, corticosteroids also suppress autoantibody formation. The serum of actively spreading vitiligo patients who received oral corticosteroids and showed improvement had a decrease in complement-mediated cytotoxicity by melanocyte autoantibodies and a reduced antibody titer to the melanocyte surface antigen. ${ }^{19}$

\section{Indications}

Systemic corticosteroids are the first-line treatment when a patient presents with rapidly progressive vitiligo. It helps not only in halting progression of the disease, but also induces repigmentation as normal melanocytes from the periphery of the lesions or perifollicular area take over, once the process of melanocyte destruction is arrested. Assessment of treatment response is important and since the patient is not always sure whether new lesions have appeared or not since corticosteroid initiation, a baseline photographic record must be taken before starting treatment and periodically throughout treatment, preferably at 4 -week intervals. The time taken for disease activity to stop is variable and may range from a few weeks to months. Once stability is achieved (defined as no new lesion formation and no extension of existing lesions), one must taper the corticosteroids slowly and add other modalities for augmentation of repigmentation.

Patients with extensive slowly progressive vitiligo (body surface area more than $10 \%$ with the continuous appearance of new lesions or slow extension of existing lesions) may also benefit from systemic corticosteroids as this suggests "simmering" autoimmune activity that may be long-term. Besides maintenance therapy, episodic treatment for 8-12 weeks at a time can also be added whenever there is relapse.

For example, systemic corticosteroids can also be added to photochemotherapy or phototherapy when there is transient fluctuation or worsening of disease activity. It can also be an adjuvant to surgery in both segmental and nonsegmental vitiligo.20,21

It is not advisable to use corticosteroid monotherapy in segmental vitiligo because segmental vitiligo is known to stabilize after some time. The initial period of progression 
may be rapid in segmental vitiligo, but nearly all lesions stabilize and remain confined to one part of the body. Hence use of corticosteroids may not be advisable. Patients with stable vitiligo will also not benefit from corticosteroids because they are not simply repigmentation agents; rather their efficacy comes from their effects on active disease. Universal or nearuniversal vitiligo is also not an indication for the use of corticosteroids since treatment is usually depigmentation of residual pigmented areas.

\section{Modes of administration}

Systemic corticosteroids given orally are effective in halting progression of vitiligo and producing repigmentation. However, the long treatment duration required in vitiligo raises concern about the long-term cumulative side effects of corticosteroids. Daily doses or alternate day dosing is thereby avoided in vitiligo. Therefore, oral mini pulse therapy (OMP) is preferred for the planned long-term maintenance therapy for 6 months or beyond. ${ }^{9} \mathrm{OMP}$ refers to giving a higher than the usual daily dose of corticosteroid on two consecutive days a week and leaving the remaining five days treatment-free. A long-acting corticosteroid is preferred so that the effect lasts for about 3 days at a time. Corticotropin and cortisol levels fall rapidly after the second dose of drug, but returns to baseline before the next dexamethasone pulse.22 Hence, it is preferred to give a long-acting corticosteroid on two consecutive days in a week rather than once in three days to give the hypothalamicpituitary-adrenal axis (HPA) axis time to revert back to normal before the next dose. The standard regimen is to give oral betamethasone $5 \mathrm{mg}$ ( 5 of the $1 \mathrm{mg}$ betamethasone tablets) after breakfast on Saturday and Sunday (or any other two consecutive days) every week. ${ }^{9}$ Dexamethasone pulse has been used in doses of $5 \mathrm{mg}$ or $10 \mathrm{mg}$ in this manner.22,23 Betamethasone pulse has been given as an adjunct with surgery. ${ }^{24}$ Besides this standard regimen, there can be variations in OMP. If no response is seen, the dose can be increased from $5 \mathrm{mg}$ upto $7.5 \mathrm{mg}$. In children, dose is reduced from $5 \mathrm{mg}$ to $2.5-4 \mathrm{mg}$. Additional immunosuppressants, for example, cyclophosphamide and azathioprine, may also be added to the therapeutic regimen.

Methylprednisolone ${ }^{25}$ has been used intravenously as high dose pulse therapy given on 3 consecutive days in a month but should not be considered as first-line treatment. Daily low dose prednisolone has also been used in tapering doses to discontinuation. ${ }^{26,27}$

The intramuscular route is not preferred as it is painful, and the level of drug in the circulation is unpredictable and good results are achieved by oral administration.

Monitoring of weight, fasting blood sugar, and blood pressure every month is necessary for any patient on systemic corticosteroids. Baseline and periodic photographs should also be done to monitor treatment response and repigmentation.

\section{Side effects and contraindications}

Systemic corticosteroids are known to have a variety of adverse effects. The predominant side effects are described in Table 2. However, these adverse effects do depend on dose, duration of treatment, and individual susceptibility. All patients do not develop the same adverse effects. Therefore, clinical evaluation and monitoring is the best way to ascertain whether adverse effects are minimal or serious, and reversible or irreversible. Minimal and reversible side effects are acceptable for continuing treatment. 


\begin{tabular}{|l|l|}
\hline \multicolumn{1}{|c|}{ System Affected } & \multicolumn{1}{c|}{ Side Effects } \\
\hline HPA Axis & Steroid withdrawal syndrome, Addisonian crisis \\
\hline Metabolic & $\begin{array}{l}\text { Hyperglycemia, increased appetite and weight gain, } \\
\text { hypertension, congestive heart failure, hypokalemia, } \\
\text { hypertriglyceridemia, cushingoid changes, menstrual } \\
\text { irregularities }\end{array}$ \\
\hline Bone & Osteoporosis, osteonecrosis, hypocalcemia \\
\hline Gastrointestinal & $\begin{array}{l}\text { Peptic ulcer disease, bowel perforation, fatty liver changes, } \\
\text { esophageal reflux, nausea, vomiting }\end{array}$ \\
\hline Ocular & Cataracts, glaucoma, infections \\
\hline Psychiatric & Psychosis, agitation, depression \\
\hline Neurological & Pseudotumor cerebri, peripheral neuropathy \\
\hline Muscular & Myopathy \\
\hline Cutaneous & $\begin{array}{l}\text { Delayed wound healing, acneiform eruption, purpura, } \\
\text { cutaneous infections, telogen effluvium, hirsutism, acanthosis } \\
\text { nigricans }\end{array}$ \\
\hline
\end{tabular}

Table 2. Side Effects of corticosteroids ${ }^{10}$

The primary consideration in treating vitiligo is careful patient selection in order to maximize response to vitiligo while minimizing adverse effects. The other concern is determination of proper dose and dosing schedule.

Side effects can be dose- or duration-dependent. Dose-dependent side effects of corticosteroids are hyperglycemia, hyperlipidemia, peptic ulcer disease, and psychosis. Duration-dependent changes are hypertension, cushingoid changes, growth impairment, osteoporosis, osteonecrosis and opportunistic infections. ${ }^{10}$ Steroid withdrawal syndrome is observed when systemic corticosteroids given at high doses are abruptly stopped and it presents as vague symptoms of lethargy, generalized weakness and myalgia. Abrupt withdrawal of the corticosteroid does not give enough time for the suppressed HPA axis to recover. It is observed with doses higher than $20-30 \mathrm{mg}$ of prednisolone. Duration of treatment is not a predictable causative factor. ${ }^{28}$ High dose of corticosteroid can also lead to extreme suppression of adrenals (Addisonian crisis) manifesting as hypotension and extreme weakness. Cushingoid changes occur with long term treatment and present as puffiness of face, moon facies, buffalo hump, striae, increased hair growth on face, skin atrophy, increased abdominal girth with thin extremities and others. Growth impairment and opportunistic infections can be decreased by avoiding daily administration either by using alternate day dosing or OMP. Oral mini pulse therapy has fewer side effects (e.g., moon facies, weight gain, acne) compared to daily dosing as reviewed in a meta-analysis. ${ }^{29}$ Betamethasone and dexamethasone can be used interchangeably as the potency and half-life are almost same. When compared to other corticosteroids, deflazacort is considered to have relatively fewer cases of growth retardation in children, osteoporosis, weight gain and HPA axis suppression. ${ }^{11}$

Absolute contraindications to use of systemic corticosteroids are very few, and include such conditions as systemic fungal infections and herpes simplex keratitis. Relative contraindications are congestive heart failure, human immunodeficiency virus (HIV), psychosis, active peptic ulcer disease, active tuberculosis, and septicemia. Well-controlled diabetes and hypertension are not contraindications, but lower doses should be used, the 
concomitant disease must be adequately treated, and the patient should be monitored frequently.

There are various approaches to minimize the side effects of corticosteroids. First is to give corticosteroids only as long as disease activity is present and the taper it slowly (over a period of 3-6 months) when no indication of new lesions are present. The oral mini pulse therapy regimen has significantly less side effects compared to typical daily dosing of corticosteroids. An important finding is the lack of prolonged HPA axis suppression with OMP therapy. ${ }^{22}$ Furthermore, higher doses of dexamethasone $(10 \mathrm{mg})$ do not seem to provide any additional benefit to $5 \mathrm{mg}$ betamethasone. ${ }^{9,}{ }^{22}$ Choosing corticosteroids with less risk of mineralocorticoid-type effects (i.e., dexamethasone, betamethasone) or one with supposedly fewer side effects (i.e., deflazacort) can be beneficial for all patients. Calcium supplementation, salt restriction, proper diet, daily exercise and use of antacids can minimize risk of side effects. Regular monitoring of weight, fasting blood sugar, and blood pressure will help in early detection of side effects if they occur.

\section{Safety in children and pregnancy}

The decision to treat progressive vitiligo in children below 4 years of age can be rather difficult as limited data is available for this population. It is suggested that oral mini pulse therapy be modified (i.e., $1 \mathrm{mg}$ of dexamethasone or betamethasone given for every $10 \mathrm{~kg}$ ) and used.

Corticosteroids have been safely used in pregnancy and are classified as category C drugs (animal reproduction studies have shown an adverse effect on the fetus and there are no adequate and well-controlled studies in humans, but potential benefits may warrant use of the drug in pregnant women despite potential risks). However, it is best to reserve its use in pregnancy when the benefits of treatment outweigh the risk of side effects. Pregnancy is known to precipitate and aggravate vitiligo. However, vitiligo being a non-life threatening condition, a patient may defer any type of treatment, including corticosteroids.

\section{Efficacy of corticosteroids in vitiligo}

Worldwide, there are few studies published documenting the efficacy and safety of corticosteroids in vitiligo. The available reports are primarily case series and there is a lack of well validated randomized controlled trials.

\subsection{Oral mini pulse therapy}

Pasricha et al evaluated the effect of five different regimens in causing repigmentation and stopping disease activity for four months and noted that regimens using corticosteroids were superior to those excluding corticosteroids. Improvement with $3 \mathrm{mg}$ betamethasone orally on alternate days combined with levamisole and topical fluocinolone, $2 \mathrm{mg}$ betamethasone orally alternating with $20 \mathrm{mg}$ 8-methoxypsoralen and sun exposure, and oral mini pulse consisting of $5 \mathrm{mg}$ betamethasone orally twice a week combined with $50 \mathrm{mg}$ cyclophosphamide daily orally was $87.5 \%, 85 \%$ and $90.9 \%$ respectively, while levamisole $150 \mathrm{mg}$ two days a week showed improvement in $53.8 \%$. Addition of topical fluocinolone acetonide to levamisole showed better response in $81.8 \% .8$

In a study on forty patients by Pasricha and Khaitan, $5 \mathrm{mg}$ betamethasone as a single oral dose after breakfast on 2 consecutive days per week was found effective in halting disease 
progression in $89 \%$ patients within 1-3 months and inducing variable spontaneous repigmentation in patients with extensive and rapidly progressive disease. ${ }^{9}$ Only 2 patients did not show response initially, but once the dose was increased from $5 \mathrm{mg}$ to $7.5 \mathrm{mg}$, there was notable improvement. Side effects were seen in $23 \%(17)$ patients, such as weight gain (5), mild headache (2), transient general weakness (2), bad taste in mouth (3), acne (2) and mild puffiness, perioral dermatitis, as well as herpes zoster, glaucoma and amenorrhea, of which were reported in one patient each. Kanwar et al ${ }^{23}$ and Radakovic-Fijan et al22 also reported efficacy of dexamethasone oral mini pulse therapy in arresting progression of rapidly spreading vitiligo in $43.8 \%$ and $88 \%$, respectively. The difference in response in this study by Kanwar et al and the one conducted by Pasricha and Khaitan could be because of the higher proportion of patients with segmental vitiligo and shorter duration of treatment in the Kanwar et al study.

OMP has been found to be effective in patients with unstable generalized and acrofacial vitiligo in a randomized double-blind placebo-controlled randomized prospective study. ${ }^{30}$

Prednisolone has been used as once a week oral dose of $2 \mathrm{mg} / \mathrm{kg}$ in 50 patients with extensive and rapidly spreading vitiligo. The disease progression was arrested in $93 \%$ and repigmentation observed in $88 \%$ with only 3 patients developing side effects. ${ }^{31}$ Methylprednisolone $0.8 \mathrm{mg} / \mathrm{kg}$ given as OMP along with topical fluticasone in children for 6 months was safe and effective in arresting disease in $90 \%$ and producing variable repigmentation in $65 \% .32$

OMP in combination with other standard vitiligo treatments has also been studied. Betamethasone has been used as an adjunct to surgery for treatment of stable vitiligo patches in patients who did not respond to their first surgical.24 It has also been combined with phototherapy and photochemotherapy. Dexamethasone OMP has been found effective and safe with psoralen with solar ultraviolet light (PUVAsol) in fast spreading vitiligo.30 $\mathrm{OMP}$ has been safely used in children and was more effective than 8-methoxypsoralen alone. ${ }^{33}$ Combination of narrowband UVB (NB-UVB) with OMP has been compared with psoralen and ultraviolet A (PUVA) photochemotherapy with OMP. Disease progression is arrested in both, but repigmentation was better and earlier with NB-UVB. ${ }^{34}$

Immunosuppressants can also be added to OMP. A comparison study of three regimens: (a) OMP with daily cyclophosphamide, (b) a 3-day dexamethasone-cyclophosphamide pulse given monthly, and (c) daily cyclophosphamide and dexamethasone-cyclophosphamide pulse given only on one day in a month showed that disease activity was arrested by all three modalities but repigmentation up to $75 \%$ was seen in $71 \%, 62 \%$, and $53 \%$, respectively. The higher response with OMP may be because weekly dosing may provide better immunosuppression in the context of vitiligo as compared to monthly dosing in pulse therapy. ${ }^{35}$ Weight gain was more frequent with OMP, but otherwise all three regimens had a similar frequency of side effects. ${ }^{35}$

\subsection{Daily corticosteroids}

In everyday practice, corticosteroids are primarily administered daily at doses individualized for each specific patient. Published studies are based on prednisolone given at $0.3 \mathrm{mg} / \mathrm{kg}$ as a single oral dose after breakfast initially for the first 2 months. The dosage is then reduced to half the initial dose during at the third month and halved again in at the fourth month of treatment. With this regimen, Kim et al noted that disease progression was halted in $87.7 \%$ of patients and in $70.4 \%$, repigmentation was also seen. ${ }^{26}$ In a study by Banerjee et al, disease 
progression was arrested in $90 \%$ and repigmentation was seen in $76 \% .27$ The side effects observed in both studies were not serious, and did not warrant a change in dose or dosing schedule. Disease was also reported to completely subside after discontinuing treatment in addition to other positive observations; this suggests that low dose corticosteroids given for 4 months may be reasonably safe and effective treatment option.

\subsection{Intravenous pulse therapy}

Methylprednisolone $(8 \mathrm{mg} / \mathrm{kg})$ given intravenously on three consecutive days a month was used in a study as pulse therapy in 14 patients with generalized vitiligo. Eighty-five percent of the patients who presented with progressive disease showed cessation of disease progression and $71 \%$ of these patients had repigmentation. None of the six patients presenting with static disease showed any repigmentation. The therapy was well tolerated in all but one patient who developed intermittent arterial hypertension during therapy. ${ }^{36}$

Most studies indicate that systemic corticosteroids are helpful in halting disease progression in rapidly spreading vitiligo and inducing spontaneous repigmentation. However their role in static vitiligo is not well documented. As of now, corticosteroids can be recommended for rapidly spreading vitiligo for a short duration to halt disease activity, but it should not be used for repigmentation in static vitiligo, even if disease is extensive. Areas that require further research is the risk of osteoporosis with corticosteroids and the efficacy of calcium supplementation. Studies with longer follow-up periods are required to evaluate the potential for relapse. Deflazacort needs to be evaluated as a relatively safer corticosteroid option for vitiligo since its use in vitiligo is very limited. Head-to-head comparative studies between betamethasone, prednisolone, and deflazacort may be useful for determining the optimal agent for treatment.

\section{Conclusion}

There seems to be a definite role for systemic corticosteroids in the treatment of vitiligo. The types of vitiligo in which benefits have been observed are: (a) rapidly progressing vitiligo, (b) slowly progressing vitiligo with extensive involvement and frequent exacerbation, (c) frank inflammatory vitiligo, (d) patients with direct or indirect evidence of autoimmune disease (e.g., high titers of thyroid autoantibodies), (d) recalcitrant static vitiligo with exacerbation, and (e) rescue therapy. Corticosteroids should not usually be considered for universal vitiligo, segmental vitiligo or focal limited vitiligo.

Although daily or alternate day oral corticosteroids may be treatment options, OMP therapy seems to be the best regimen for long-term use with minimal side effects. The duration of OMP should be at least 6 months or longer and tapering to discontinuation should be slow and step-wise. When a patient is on any regimen that includes systemic corticosteroids, regular and periodic monitoring must be done to assess the risk-to-benefit ratio of treatment and to evaluate efficacy. Further study is needed to provide more definitive evidence for systemic corticosteroid use in vitiligo as well as the long-term implications of treatment.

\section{References}

[1] Majumdar PP. Genetics and prevalence of vitiligo vulgaris. In: Hann SK, Nordlund JJ, Editors. Vitiligo. 2000, Blackwell Science Ltd. p. 18-20. 
[2] Mattoo SK, Handa S, Kaur I, Gupta N, Malhotra R. Psychiatric morbidity in vitiligo: prevalence and correlates in India. J Eur Acad Dermatol Venereol. 2002; 16: 573-8.

[3] Linthorst Homan MW, Spuls PI, de Korte J, Bos JD, Sprangers MA, van der Veen JP. The burden of vitiligo: patient characteristics associated with quality of life. J Am Acad Dermatol. 2009; 61: 411-20.

[4] Pasricha JS, Khaitan BK. Drugs for vitiligo. New Age International (P) limited, 1996, New Delhi. p. 37-43.

[5] Farah FS, Kurban AK, Chaglassian HT. The treatment of vitiligo with psoralens and triamcinolone by mouth. Br J Dermatol 1967; 79: 89-91.

[6] Gokhale BB, Gokhale TB. Corticotrophin and vitiligo (preliminary observations). Br J Dermatol. 1976; 95: 329.

[7] Imamura S, Tagami H. Treatment of vitiligo with oral corticosteroids. Dermatologica. 1976; 153: 179-85.

[8] Pasricha JS, Seetharam KA, Dashore A. Evaluation of five different regimes for the treatment of vitiligo. Indian J Dermatol Venereol 1989; 55: 18-21.

[9] Pasricha JS, Khaitan BK. Oral mini-pulse therapy with betamethasone in vitiligo patients having extensive or fast-spreading disease.Int J Dermatol. 1993; 32: 753-7.

[10] Wolverton SE. Systemic corticosteroids. In :Wolverton SE. Comprehensive dermatologic drug therapy. 2nd edition. Saunders Elseviers, Philadelphia. 2007. p 127-61.

[11] Joshi N, Rajeshwari K. Deflazacort. J Postgrad Med 2009; 55: 296-300.

[12] Abdel-Naser MB, Krüger-Krasagakes S, Krasagakis K, Gollnick H, Abdel-Fattah A, Orfanos CE. Further evidence for involvement of both cell mediated and humoral immunity in generalized vitiligo. Pigment Cell Res. 1994; 7: 1-8.

[13] Ahn SK, Choi EH, Lee SH, Won JH, Hann SK, Park YK. Immunohistochemical studies from vitiligo--comparison between active and inactive lesions. Yonsei Med J. 1994; 35: 404-10.

[14] Abdel-Naser MB, Ludwig WD, Gollnick H, Orfanos CE. Nonsegmental vitiligo: decrease of the CD45RA+ T-cell subset and evidence for peripheral T-cell activation. Int J Dermatol. 1992; 31: 321-6.

[15] Ogg GS, Rod Dunbar P, Romero P, Chen JL, Cerundolo V. High frequency of skinhoming melanocyte-specific cytotoxic T lymphocytes in autoimmune vitiligo. J Exp Med. 1998; 188: 1203-8.

[16] Wańkowicz-Kalińska A, van den Wijngaard RM, Tigges BJ, Westerhof W, Ogg GS,etal. Immunopolarization of CD4+ and CD8+ T cells to Type-1-like is associated with melanocyte loss in human vitiligo. Lab Invest. 2003; 83: 683-95.

[17] van den Boorn JG, Konijnenberg D, Dellemijn TA, van der Veen JP, Bos JD, Melief CJ, Vyth-Dreese FA, Luiten RM. Autoimmune destruction of skin melanocytes by perilesional T cells from vitiligo patients. J Invest Dermatol. 2009; 129: 2220-32.

[18] Klarquist J, Denman CJ, Hernandez C, Wainwright DA, Strickland FM, Overbeck A, etal. Reduced skin homing by functional Treg in vitiligo. Pigment Cell Melanoma Res. 2010; 23: 276-86.

[19] Hann SK, Kim HI, Im S, Park YK, Cui J, Bystryn JC. The change of melanocyte cytotoxicity after systemic steroid treatment in vitiligo patients. J Dermatol Sci. 1993; 6: 201-5.

[20] Lee DY, Choi SC, Lee JH. Generalized vitiligo treated by combination therapy of epidermal graft and systemic corticosteroid. Clin Exp Dermatol. 2009; 34: 838. 
[21] Lee DY, Lee KJ, Choi SC, Lee JH. Segmental vitiligo treated by the combination of epidermal grafting and systemic corticosteroids. Dermatol Surg. 2010; 36: 575-6.

[22] Radakovic-Fijan S, Fürnsinn-Friedl AM, Hönigsmann H, TanewA. Oral dexamethasone pulse treatment for vitiligo. J Am Acad Dermatol. 2001; 44: 814-7.

[23] Kanwar AJ, Dhar S, Dawn G. Oral minipulse therapy in vitiligo.Dermatology1995; 190: 251-2.

[24] Mulekar SV. Stable vitiligo treated by a combination of low-dose oral pulse betamethasone and autologous, noncultured melanocyte-keratinocyte cell transplantation. Dermatol Surg. 2006; 32: 536-41.

[25] Seiter S, Ugurel S, Pföhler C, Tilgen W, Reinhold U. Successful treatment of progressive vitiligo with high-dose intravenous methylprednisolone 'pulse' therapy. Dermatology. 1999; 199: 261-2.

[26] Kim SM, Lee HS, Hann SK. The efficacy of low-dose oral corticosteroids in the treatment of vitiligo patients. Int J Dermatol 1999; 38: 546-50.

[27] Banerjee K, Barbhuiya JN, Ghosh AP, Dey SK, Karmakar PR. The efficacy of low-dose oral corticosteroids in the treatment of vitiligo patient. Indian J Dermatol Venereol Leprol. 2003; 69: 135-7.

[28] Daly JR, Myles AB, Bacon PA, Beardwell CG, Savage O. pituitary adrenal function during corticosteroid withdrwal in rheumatoid arthritis. Ann Theum Dis 1967; 26; 18-25.

[29] Njoo MD, Spuls PI, Bos JD, Westerhof W, Bossuyt PM. Non-surgical repigmentation therapies in vitiligo. Meta-analysis of the literature. Arch Dermatol. 1998; 134: 1532-40.

[30] Paul M et al. OMP steroid therapy in the treatment of unstable vitiligo-a double blind placebo controlled randomized prospective study. Presented at $26^{\text {th }}$ Annual Conference of IADVL, 1998.

[31] Gupta RR. Modified oral mini pulse steroid therapy in unstable vitiligo-A novel once a week regimen. Presented at 29th Annual Conference of IADVL, 2001.

[32] Imran $M$ et al. Childhood vitiligo: Response to methylprednisolone OMP therapy and topical fluticasone preparation. Indian J Dermatol 2010; 54: 124-7.

[33] Nigam PK. Clinical and oral minipulse therapy evaluation in childhood vitiligo. Presented at 32th Annual Conference of IADVL, 2004.

[34] Rath $\mathrm{N}$ et al. PUVA vs NB(UVB) with OMP: efficacy in progressive vitiligo. Presented at 32nd Annual Conference IADVL, 2004.

[35] Singh YL, Khaitan BK, Ramam M, Pasricha JS. A comparative study of the effect of three regimens comprising of corticosteroids and cyclophosphamide for the treatment of vitiligo. Thesis submitted to AIIMS, June 1999 (unpublished).

[36] Seiter S, Ugurel S, Tilgen W, Reinhold U. Use of high-dose methylprednisolone pulse therapy in patients with progressive and stable vitiligo. Int J Dermatol. 2000; 39: 624-7. 


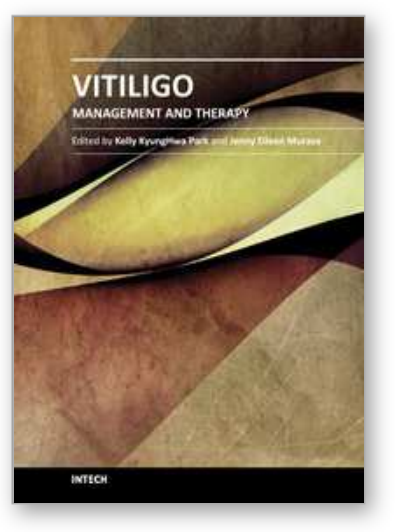

\author{
Vitiligo - Management and Therapy \\ Edited by Dr. Kelly KyungHwa Park
}

ISBN 978-953-307-731-4

Hard cover, 174 pages

Publisher InTech

Published online 14, December, 2011

Published in print edition December, 2011

Vitiligo: Management and Therapy is a practical guide to vitiligo that reflects current research related to the fundamentals of vitiligo and its management. Vitiligo experts and researchers from all over the world have contributed to this text, accounting for its comprehensive nature and diverse array of topics. The recent advances in medicine and technology have led to a better understanding of the disease and have broadened available treatment options. The essentials are captured in this book and are complemented by useful clinical photographs and reference tables. This concise tool will serve as an invaluable resource for clinicians in daily practice.

\title{
How to reference
}

In order to correctly reference this scholarly work, feel free to copy and paste the following:

Binod K. Khaitan and Sushruta Kathuria (2011). Systemic Corticosteroids in Vitiligo, Vitiligo - Management and Therapy, Dr. Kelly KyungHwa Park (Ed.), ISBN: 978-953-307-731-4, InTech, Available from:

http://www.intechopen.com/books/vitiligo-management-and-therapy/systemic-corticosteroids-in-vitiligo

\section{INTECH}

open science | open minds

\section{InTech Europe}

University Campus STeP Ri

Slavka Krautzeka 83/A

51000 Rijeka, Croatia

Phone: +385 (51) 770447

Fax: +385 (51) 686166

www.intechopen.com

\section{InTech China}

Unit 405, Office Block, Hotel Equatorial Shanghai

No.65, Yan An Road (West), Shanghai, 200040, China

中国上海市延安西路65号上海国际贵都大饭店办公楼 405 单元

Phone: +86-21-62489820

Fax: +86-21-62489821 
(C) 2011 The Author(s). Licensee IntechOpen. This is an open access article distributed under the terms of the Creative Commons Attribution 3.0 License, which permits unrestricted use, distribution, and reproduction in any medium, provided the original work is properly cited. 